\title{
High concordance between hippocampal transcriptome of the mouse intra-amygdala kainic acid model and human temporal lobe epilepsy
}

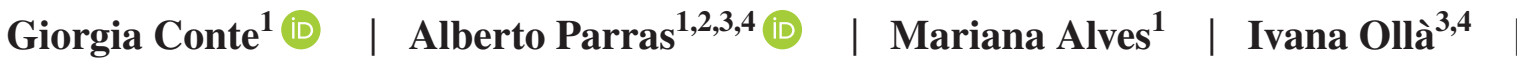 \\ Laura De Diego-Garcia $^{1}$ | Edward Beamer ${ }^{1,5}$ | Razi Alalqam $^{1}$ | Alejandro Ocampo ${ }^{2}$ | \\ Raúl Mendez $^{6,7}$ | David C. Henshall ${ }^{1,8}$ (D) | José J. Lucas ${ }^{3,4}$ | Tobias Engel ${ }^{1,8}$ (D)
}

${ }^{1}$ Department of Physiology and Medical Physics, Royal College of Surgeons in Ireland, University of Medicine and Health Sciences, Dublin, Ireland

${ }^{2}$ Department of Biomedical Sciences, Faculty of Biology and Medicine, University of Lausanne, Lausanne, Switzerland

${ }^{3}$ Severo Ochoa Center for Molecular Biology-CBMSO, CSIC/UAM, Madrid, Spain

${ }^{4}$ Networking Research Center on Neurodegenerative Diseases-CiberNed, Carlos III Institute of Health, Madrid, Spain

${ }^{5}$ Department of Science and Engineering, John Dalton Building, All Saints Campus, Metropolitan University, Manchester, UK

${ }^{6}$ Institute for Research in Biomedicine, Barcelona Institute of Science and Technology, Barcelona, Spain

${ }^{7}$ Catalan Institution for Research and Advanced Studies, Barcelona, Spain

${ }^{8}$ FutureNeuro, Science Foundation Ireland Research Centre for Chronic and Rare Neurological Diseases, Royal College of Surgeons in Ireland, University of Medicine and Health Sciences, Dublin, Ireland

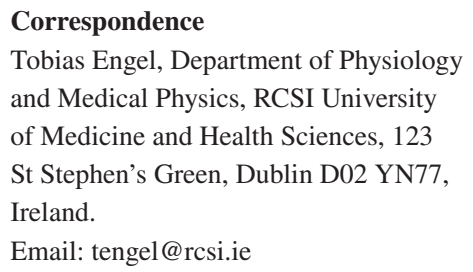

Email: tengel@rcsi.ie

\begin{abstract}
Objective: Pharmacoresistance and the lack of disease-modifying actions of current antiseizure drugs persist as major challenges in the treatment of epilepsy. Experimental models of chemoconvulsant-induced status epilepticus remain the models of choice to discover potential antiepileptogenic drugs, but doubts remain as to the extent to which they model human pathophysiology. The aim of the present study was to compare the molecular landscape of the intra-amygdala kainic acid model of status epilepticus in mice with findings in resected brain tissue from patients with drug-resistant temporal lobe epilepsy (TLE).
\end{abstract}

Methods: Status epilepticus was induced via intra-amygdala microinjection of kainic acid in C57BL/6 mice, and gene expression was analyzed via microarrays in hippocampal tissue at acute and chronic time-points. Results were compared to reference datasets in the intraperitoneal pilocarpine and intrahippocampal kainic acid model and to human resected brain tissue (hippocampus and cortex) from patients with drug-resistant TLE.

Results: Intra-amygdala kainic acid injection in mice triggered extensive dysregulation of gene expression that was $\sim 3$-fold greater shortly after status epilepticus (2729 genes) when compared to epilepsy (412). Comparison to samples from patients with TLE revealed a particularly high correlation of gene dysregulation during established epilepsy. Pathway analysis found suppression of calcium signaling to be highly conserved across different models of epilepsy and patients. cAMP response elementbinding protein (CREB) was predicted as one of the main upstream transcription factors regulating gene expression during acute and chronic phases, and inhibition of CREB reduced seizure severity in the intra-amygdala kainic acid model.

Significance: Our findings suggest the intra-amygdala kainic acid model faithfully replicates key molecular features of human drug-resistant TLE and provides potential

Giorgia Conte and Alberto Parras contributed equally. 


\section{Funding information}

Health Research Board, Grant/Award Number: HRA-POR-2015-1243; Science

Foundation Ireland, Grant/Award Number: 16/RC/3948 and 17/CDA/4708; H2020

Environment, Grant/Award Number: 753527, 766124 and 796600; Centro de Investigación Biomédica en Red de Enfermedades Neurodegenerativas, Grant/ Award Number: PI2015-2/06-3 and PI2018/06-1; Ministerio de Economía y Competitividad, Grant/Award Number: SAF2015-65371-R; Ministerio de Ciencia, Innovación y Universidades, Grant/Award Number: RTI2018-096322-B-I00 rational target approaches for disease-modification through new insights into the unique and shared gene expression landscape in experimental epilepsy.

\section{K EYWORDS}

calcium signaling, CREB, epilepsy, mouse model, status epilepticus, transcriptome

\section{1 | INTRODUCTION}

A major challenge in epilepsy is the lack of adequate treatment, with $>30 \%$ of patients remaining resistant to currently available antiseizure drugs (ASDs). ${ }^{1,2}$ Moreover, ASDs may cause severe adverse effects, and critically, current pharmacological treatment remains symptomatic without significantly altering the course of the disease. ${ }^{3}$ The most common drug-refractory form of epilepsy in adults is temporal lobe epilepsy (TLE) involving different structures within the limbic system, including the amygdala and hippocampus. ${ }^{4}$ Pathological processes occurring during epileptogenesis include structural and functional changes such as ongoing neurodegeneration and reorganization of neural networks. ${ }^{5}$ Mounting data obtained via gene expression profiling suggest these processes are driven in part by large-scale changes in the gene expression landscape within the brain. ${ }^{6-14}$

Whereas animal models of acute seizures (eg, pentylenetetrazol, maximal electroshock) have been successful in the identification of ASDs, the identification of antiepileptogenic drugs and drugs to treat refractory epilepsy most likely requires different models that phenocopy the chronic stage of the disease. ${ }^{15}$ The translational value of a model depends, however, on there being extensive homology with the human pathophysiology, or we risk developing ineffective treatments. Currently, rodent models of status epilepticus (SE) remain the preferred method for generating drug-resistant epilepsy. The extent to which these match the molecular landscape of human refractory TLE remains uncertain. The intra-amygdala kainic acid (IAKA) model of focal onset SE in mice produces unilateral pathology and drug-resistant epilepsy after a short latent period. ${ }^{16,17}$ The model is increasingly used for studying mechanisms of epileptogenesis and the testing of novel antiepileptogenic drugs. ${ }^{17-23}$ Whereas changes in the transcriptome have been analyzed shortly following SE in the model, ${ }^{24,25}$ global changes in gene expression during chronic epilepsy and whether alterations in gene expression reflect changes occurring in patients remain to be established.

\section{Key Points}

- More genes show expression changes shortly following intra-amygdala kainic acid-induced status epilepticus when compared to established epilepsy

- The intra-amygdala kainic acid mouse model closely mimics the gene expression landscape in the brain of patients with temporal lobe epilepsy

- Suppressed calcium signaling in the brain is a common feature across experimental models of epilepsy and patients with temporal lobe epilepsy

- CREB is a major upstream transcription factor during early changes following status epilepticus and once epilepsy is established

Here, we compared the gene expression profile in the IAKA mouse model with reference data from patients with drug-resistant TLE and two other models of SE as comparators. Our results reveal excellent molecular concordance between the IAKA model and human TLE and identify potential targets for disease-modifying treatments.

\section{2 | MATERIALS AND METHODS}

Reagents were purchased from Sigma-Aldrich if not stated otherwise.

\subsection{Animal model of SE}

Animal experiments were performed in accordance with the principles of the European Communities Council Directive (2010/63/EU) and approved by the Research Ethics Committee of the Royal College of Surgeons in Ireland (RCSI; REC1322, 842) and the Irish Health Products 
Regulatory Authority (AE19127/P038). Experiments were carried out in 8- to 12-week-old C57B1/6 male mice bred at RCSI. SE was induced in fully awake mice via a microinjection of kainic acid (KA; $0.3 \mu \mathrm{g}$ in $0.2 \mu \mathrm{L}$ phosphate-buffered saline [PBS]) into the basolateral amygdala. Vehicle-injected control animals received $0.2 \mu \mathrm{L}$ PBS. The anticonvulsant lorazepam $(6 \mathrm{mg} / \mathrm{kg}$; Wyetch) was delivered intraperitoneally (i.p.) 40 minutes after IAKA or vehicle to curtail seizures and reduce morbidity and mortality. Electroencephalogram (EEG) was recorded from cortical implanted electrodes (Xltek recording system; Optima Medical) starting $10 \mathrm{~min}$ utes prior to IAKA administration. ${ }^{26}$

\section{2 | EEG quantification and behavioral assessment of seizure severity during SE}

The duration of high-frequency $(>5 \mathrm{~Hz})$ and high-amplitude ( $>2 \times$ baseline; HFHA) polyspike discharges of $\geq 5$-second duration ${ }^{27}$ was counted manually, and EEG total power $\left(\mu \mathrm{V}^{2}\right)$ was analyzed via Labchart7 (ADInstruments) by integrating frequency bands from 0 to $100 \mathrm{~Hz}$. Power spectral density heat maps were generated using Labchart7 (frequency $=0-40 \mathrm{~Hz}$, amplitude $=0-50 \mathrm{mV}$ ). EEG was recorded from a total of 20 mice during three individual experiments (four mice were excluded from the EEG analysis due to mortality during SE [three vehicle-injected mice and one cAMP response elementbinding protein (CREB) inhibitor 666-15-treated mouse]). Clinical behavior was scored in a subset of 12 mice every 5 minutes for 40 minutes after IAKA according to a modified Racine scale by an observer blinded to treatment. ${ }^{28}$

\subsection{Drug treatment}

Mice received an intracerebroventricular (i.c.v.) infusion of 2 nmol of the CREB inhibitor $666-15^{29,30}$ in $2 \mu \mathrm{L}$ volume (PBS in $0.2 \%$ dimethyl sulfoxide) to reach a final concentration of $1 \mathrm{mmol} \cdot \mathrm{L}^{-1}$ in the ventricle (ventricle volume $\sim 35 \mu \mathrm{L}$ ). Mice were treated once 10 minutes before IAKA injection or 60 minutes after lorazepam administration.

\subsection{RNA isolation and microarray analysis}

Mice were euthanized 8 hours or 14 days post-SE. Ipsilateral hippocampi were quickly dissected and pooled ( $n=3$ /pooled sample). RNA was extracted using the Maxwell 16 LEV simplyRNA Tissue Kit (Promega, AS1280). RNA quantification was performed with Qubit RNA Hs Assay kit (Thermo Fisher Scientific, Q32852) and RNA integrity quality control with Agilent Bioanalyzer 2100, using RNA Nano/Pico Assay (Agilent Technologies 5067-1511, 5067-1513). cDNA library preparation and amplification were performed with WTA2 kit using 2-5 ng of total RNA as template. cDNA was amplified for 22 cycles and purified using PureLink Quick PCR Purification Kit (Invitrogen, K310001), thereby increasing the detection sensitivity. This, however, may also lead to the overrepresentation of enriched genes. Eight micrograms of cDNA from each sample was fragmented and labeled with GeneChip Mapping 250K Nsp assay kit (Affymetrix, 900753). Hybridization was performed for 16 hours at $45^{\circ} \mathrm{C} .{ }^{31,32}$ Washing and staining steps were performed in the GeneAtlas Fluidics Station (Affymetrix, 00-0079), following the specific script for Mouse MG-430 PM Arrays. Arrays were scanned with GeneAtlas Scanner, and CEL files were done using GeneAtlas software (Affymetrix). Processing of microarray samples was performed using $\mathrm{R}$ and Bioconductor. ${ }^{33}$ Raw CEL files were normalized using RMA background correction and summarization. Probeset annotation was performed using Affymetrix version na35. For each gene, a linear model was used to find significant differences between IAKA- or vehicle (PBS)-treated mice. Analysis of differential expression was performed using a linear model implemented in the R package "limma." ${ }^{34} P$ values were adjusted with the Benjamini and Hochberg correction. We considered one gene to be downregulated with an adjusted $P$ value $\leq .01$ and fold change $(\mathrm{FC})<-1.2$ and upregulated with adjusted $P$ value $\leq$ .01 and $\mathrm{FC}>1.2$ in at least one probe. If the same transcript showed opposite results for different probes, the transcript was considered to be not changed.

\section{5 $\quad$ RNA extraction and quantitative polymerase chain reaction}

RNA was extracted from ipsilateral hippocampi. ${ }^{26}$ RNA concentration was measured via a nanodrop Spectrophotometer (Thermo Fisher Scientific). Samples with an absorbance (260/180) ratio between 1.8 and 2.2 were considered acceptable. Five hundred micrograms of mRNA was used to produce cDNA by reverse transcription using SuperScript III reverse transcriptase enzyme (Invitrogen). Quantitative polymerase chain reaction (PCR) was performed using LightCycler 1.5 (Roche Diagnostics). Each reaction tube contained $2 \mu \mathrm{L}$ cDNA, $10 \mu \mathrm{L}$ SYBR Green Quantitect Reagent (Qiagen), $1.25 \mu \mathrm{mol} \cdot \mathrm{L}^{-1}$ primer pair and RNAse-free water (Invitrogen) to a final volume of $20 \mu \mathrm{L}$. Data were analyzed and normalized to the expression of $\beta$-actin. Primers are listed in Table S1.

\subsection{Comparison of gene expression changes between models}

The degree of differential expression observed between profiling studies was analyzed via the hypergeometric distribution test. This approach is particularly sensitive and robust to 
detect possible overlaps between different gene expression profiles despite experimental and biological confounding factors. ${ }^{35}$ Gene sets were compared using the representation factor (RF). RF is the number of overlapping genes (genes in common) divided by the expected number of overlapping genes drawn from two independent groups. Two gene sets were considered overlapping only when $\mathrm{RF}>2$ and $P<.05$. Gene profiling data for the pilocarpine mouse model (12 hours and 6 weeks post-SE) were obtained from Hansen et $\mathrm{al}^{10}$ and for the intrahippocampal KA (IHKA) model (6 hours and 2 weeks post-SE) from Motti et al. ${ }^{36}$ Transcriptome changes in TLE patients were previously reported in Guelfi et $\mathrm{al}^{14}$ for cortical tissue samples from 86 mesial TLE (mTLE) patients versus 75 neurologically healthy controls, and in Salman et $\mathrm{al}^{12}$ for sclerotic hippocampus versus nonspiking neocortex from 10 TLE patients. See Table S2 for the complete set of genes used.

\section{7 | Gene ontology analysis}

Pathway analysis of up- and downregulated genes (adjusted $P$ value $\leq .01$ and $\mathrm{FC}> \pm 1.2$ ) in the IAKA model ( 8 hours and 14 days post-SE) and of overlapping genes between the IAKA model and TLE patients ${ }^{12}$ was carried out using DAVID Bioinformatics Resources 6.7, KEGG pathway annotation. $^{37}$

\subsection{Ingenuity Pathway Analysis}

Analysis of upstream regulators was performed applying Ingenuity Pathway Analysis (IPA; QIAGEN) ${ }^{38}$ on genes found differentially expressed (adjusted $P$ value $<.01$ and $\mathrm{FC}> \pm 1.2$ ) in the IAKA mouse model and TLE patients. ${ }^{12,14}$

\section{9 | Data analysis}

We used 36 mice for microarray analysis, 29 mice to analyze the role of CREB during SE, and 18 mice for gene expression validation via single quantitative real-time PCR (qRT-PCR), totaling 83 mice. Statistical analysis was performed using SPSS 25.0 (IBM). Data are represented as mean \pm standard error of the mean with $95 \%$ confidence interval. Higher or lower points (outliers) are plotted individually or are not plotted. The normality of the data was analyzed by ShapiroWilk test and homogeneity of variance by Levente test. For comparison of two independent groups, unpaired two-tailed Student $t$ test (data with normal distribution), or MannWhitney-Wilcoxon or Kolmogorov-Smirnov test (nonnormal distribution) was performed. Enrichment tests were carried out by using one-sided Fisher exact test. Significance was accepted at $P<.05$.

\subsection{0 | Data availability}

Data supporting our findings are available from the corresponding authors upon reasonable request (GEO accession number GSE122228: Identification of Differentially Expressed Genes in the Hippocampus in the Intra-Amygdala KA Mouse Model of SE).

\section{\begin{tabular}{l|l}
3 & RESULTS
\end{tabular}}

\section{1 | Larger transcriptome changes following SE compared to established epilepsy in IAKA-treated mice}

mRNA was extracted from ipsilateral hippocampi of vehicle-injected control mice and mice subjected to IAKAinduced $\mathrm{SE}^{16}$ and analyzed via microarrays (total of 21549 genes). For the acute time-point, we chose 8 hours post-SE. At this time, seizure activity has ceased, EEG usually has returned to baseline levels, and the first signs of seizureinduced neurodegeneration have appeared, but without the widespread cell death observed at 24 hours post-SE. ${ }^{39}$ As a second time-point, we chose 14 days post-SE, a timepoint when all mice subjected to IAKA are normally experiencing regular spontaneous seizures. ${ }^{16,20,25}$ To reduce intersample variability, each sample analyzed was a pool of three ipsilateral hippocampi (Figure 1A). Genes were considered to be differentially expressed when adjusted $P$ value $\leq .01$ and $\mathrm{FC}<-1.2$ for downregulated and $\mathrm{FC}$ $>1.2$ for upregulated transcripts (Figure 1B, Table S3). Validation of a set of transcripts via qRT-PCR confirmed the microarray findings for Hspalb, Atf4, Ctsz, and Laptm5 (Figure S1A,B).

The greatest FC in gene expression post-SE was found for known activity-regulated transcripts, including c-Fos $(\mathrm{FC}=58.1)$ and $\operatorname{Inhb}(\mathrm{FC}=33.2)$. The most downregulated transcripts were Hes5 $(\mathrm{FC}=-6.8)$ and Gpr12 (FC $=-4.0$; Figure 1C). A similar number of genes were up- (1366) and downregulated (1363) post-SE. In contrast, during epilepsy, genes were mainly upregulated (331), with only 81 genes downregulated (Figure 1D). Furthermore, more genes showed alterations of their mRNA levels following SE (2729 genes, 12.6\%) when compared to established epilepsy (412 genes, 1.9\%; Figure 1D,E). Finally, we found a very significant enrichment of up- and downregulated genes between both time-points, indicating that gene expression changes observed post-SE are preserved during epilepsy (Figure 1F). 
Together, our results show that IAKA-induced SE leads to a unique expression profile during SE and epilepsy, with changes in mRNA levels being more prominent post-SE when compared to established epilepsy.

\section{2 | IAKA mouse model mimics the hippocampal gene expression profile of TLE in humans}

Chemoconvulsant-induced SE is one of the most common strategies to identify novel antiepileptic drugs, with KA and pilocarpine being the most frequently used. ${ }^{15}$ To establish whether gene expression changes are similar between models, we compared transcriptome profiles of the IAKA mouse model with transcriptome changes reported for other models where epilepsy is induced via SE, including the IHKA mouse model ${ }^{36}$ and the i.p. pilocarpine mouse model ${ }^{10}$ (Table S2). Of note, whereas the cholinergic agonist pilocarpine acts on muscarinic receptors including both $\mathrm{M} 1$ and $\mathrm{M} 2$ receptors, KA activates ionotropic glutamatergic KA receptors. This comparison revealed a similar expression pattern between the IAKA and i.p. pilocarpine mouse model at both time-points, post-SE and epilepsy, with expression changes being more similar during established epilepsy (Figure 2A). Notably, the IAKA and IHKA mouse model showed an almost complete overlap in gene expression post-SE and during epilepsy (Figure 2B).

Next, to determine the extent to which mRNA changes occurring in mouse models are translatable to TLE in humans, we compared transcriptome changes with recently published transcriptome changes in TLE patients. This included a study analyzing changes in sclerotic hippocampi versus neocortex of TLE patients ${ }^{12}$ and a study analyzing gene expression changes in cortical tissue from patients with mTLE $^{14}$ (Table S2). Here, we found a very strong correlation between transcriptome changes identified in the IAKA mouse model during epilepsy and changes observed in TLE patients (hippocampus and cortex; Figure 2C). Similar to the IAKA model, there was also a strong correlation of dysregulated genes between the IHKA model and TLE patients (Figure S2A). In contrast, the transcriptional profile differed greatly when comparing between human TLE and expression changes 8 hours following IAKAinduced SE (Figure S2B). Finally, although there was a good overlap among downregulated genes between pilocarpine-injected mice and TLE patients, this correlation was lost among upregulated genes. Moreover, the magnitude of enrichments was minor when compared to both KA mouse models (Figure S2C), suggesting KA mouse models reflect closer molecular changes that occur in the brain of TLE patients than the pilocarpine model. The overlap of differentially regulated genes between datasets is shown in Figure S3A,B.

\section{3 | Suppressed calcium signaling as a common feature across experimental models of epilepsy and TLE patients}

To identify pathways and functional groups of genes affected by expression changes in the IAKA mouse model, differentially expressed transcripts were analyzed by Gene Ontology (GO) terms using the bioinformatic tool DAVID. ${ }^{40}$ This revealed that post-SE upregulated genes are mainly associated with signaling pathways (eg, MAPK, PI3K-Akt) and downregulated genes with metabolism and synaptic transmission (Figure 3A, Table S4). At 14 days post-SE, when epilepsy is established, upregulated genes are highly enriched in pathways linked to waste removal (eg, phagosomes, lysosomes) and downregulated genes are mainly linked to pathways controlling synaptic transmission (Figure 3A), similar to downregulated genes post-SE (Figure 3A, Table S4).

GO term analysis of dysregulated genes shared between the IAKA mouse model (epilepsy) and TLE patients revealed a significant enrichment of genes linked to intracellular signaling (eg, PI3K-Akt, MAPK, TGF- $\beta$ ) and lysosome activity among upregulated genes, and genes associated with synaptic transmission (eg, calcium signaling, long-term potentiation) among downregulated genes (Figure 3B). Most notably, genes enriched in calcium signaling were significantly affected in all three conditions (Figure 3A,B, Table S4).

To further assess whether the enrichment of downregulated genes involved in calcium signaling is a common response across different models, GO terms of altered transcripts were analyzed in all three animal models (IAKA, IHKA, pilocarpine) and TLE patients. ${ }^{12,14}$ This confirmed calcium signaling as a common pathway suppressed in mouse models of epilepsy and TLE patients (Figure 3C, Table S4). Downregulated genes involved in calcium signaling and altered post-SE and in human TLE are shown in Table 1. Microarray results (Figure S4A,B) were confirmed via single qRT-PCR analysis of selected downregulated transcripts involved in calcium signaling either unique to IAKAinduced SE (Figure 3D) or common between SE and epilepsy (Figure 3E).

In summary, our results reveal overlap of several altered pathways between the IAKA mouse model and human TLE, particularly genes linked to calcium signaling, thereby identifying possible novel treatment targets for epilepsy.

\subsection{CREB is a major transcription factor controlling gene transcription following IAKA- induced SE and during epilepsy}

Analysis via IPA ${ }^{38}$ predicted several transcription factors to regulate gene expression changes post-SE and during epilepsy. Most notably, the majority of identified transcription 
A

\section{Experimental design}
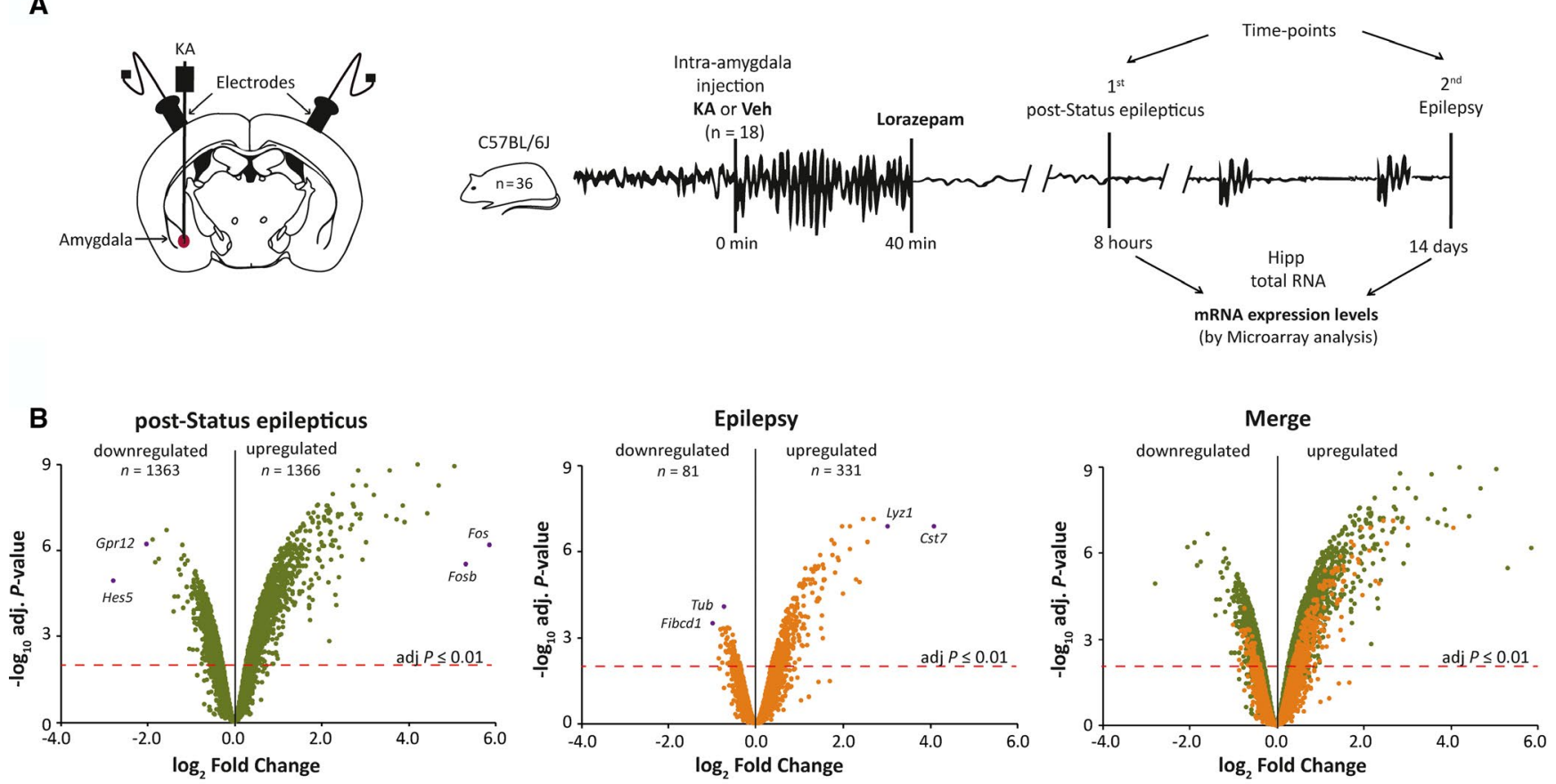

Heatmap of genes with most extreme changes $(<-1.75$ or $>4)$

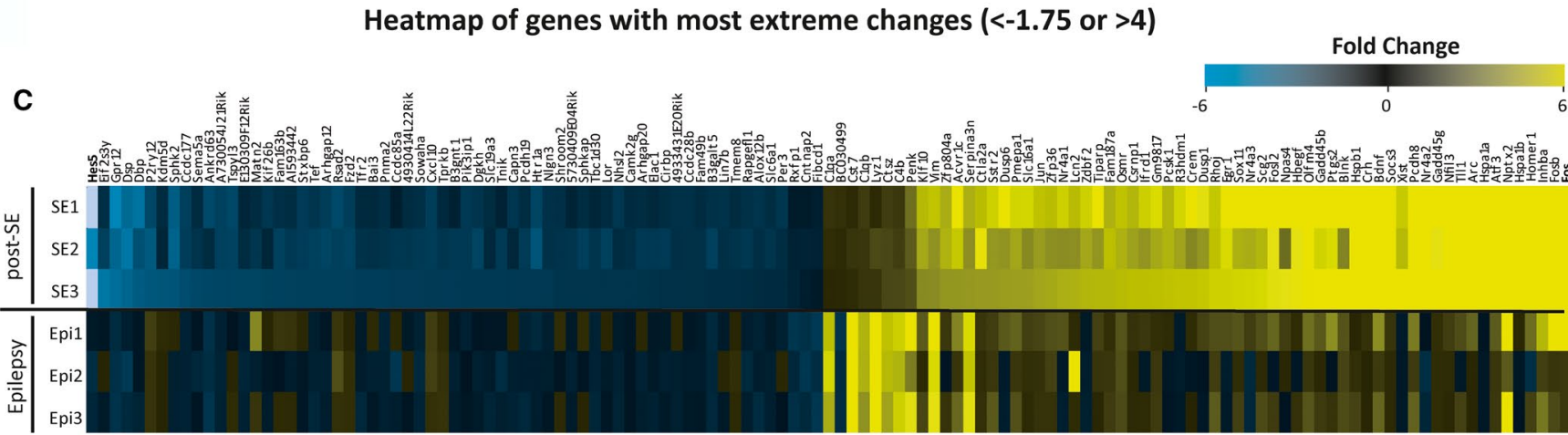

D Gene expression changes

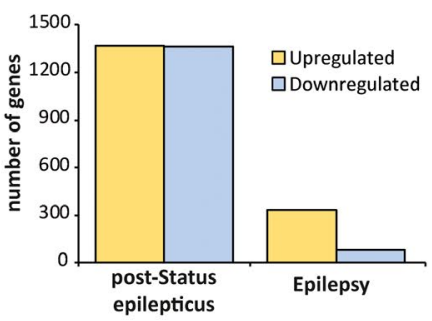

E Differentially expressed genes

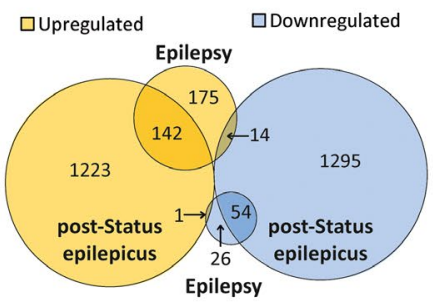

F Shared gene expression post-SE and Epilepsy

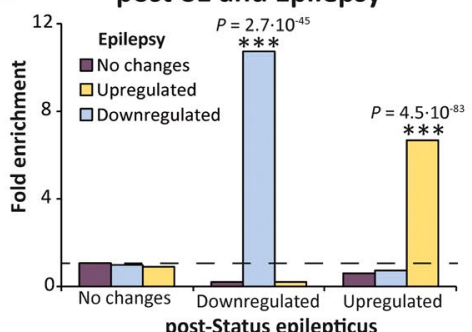

factors are unique to SE or epilepsy (eg, TRP53 and FOS post-SE), confirming the limited overlap of differently expressed genes between conditions. Some transcription factors were, however, predicted to control gene expression in both conditions, including MYC (upregulated genes postSE and during epilepsy) and CREB1, linked to both up- and downregulated genes under both conditions (Figure 4A, Table S5). Importantly, CREB1 was also determined to regulate expression of upregulated genes in both cortex $\left(P=2.1 \times 10^{-2}\right)$ and hippocampus $\left(P=2.5 \times 10^{-4}\right)$ from patients with TLE.

To test whether CREB has a role during seizure-induced pathology in the IAKA mouse model, mice were treated i.c.v. with the CREB-specific inhibitor $666-15^{30}$ (Figure 4B). Demonstrating CREB regulating the expression of genes linked to calcium signaling, both $\mathrm{CamK} 4$ and 
F I G U R E 1 Gene expression analysis after intra-amygdala kainic acid (KA)-induced status epilepticus (SE) and during epilepsy. A, Schematic showing experimental design using the intra-amygdala KA (IAKA)-induced SE mouse model. Vehicle (Veh)-injected control mice and mice subjected to IAKA were euthanized at two time-points: 8 hours post-SE (acute) and 14 days (chronic epilepsy). Each sample analyzed via microarray was a pool of three ipsilateral hippocampi (Hipp; $\mathrm{n}=9$ per group [vehicle-control and IAKA], totaling 36 mice including both post-SE and epilepsy). B, Volcano plot representing genes analyzed via microarray post-SE (left panel) and during chronic epilepsy (middle panel), and overlap of both time-points (post-SE and epilepsy, right panel). The x-axis represents the $\log _{2}$ ratio of gene expression levels and the $y$-axis the adjusted $P$ value based on $-\log _{10}$. The red dashed lines denote the significant level $(P \leq .01)$. Purple dots represent genes with the most dramatic expression changes. C, Heatmap of genes showing the most dramatic expression changes (blue indicates downregulated fold change [FC] $<-1.75$; yellow indicates upregulated FC $>4$ ), post-SE (acute; top part) and during chronic epilepsy (bottom part). D, Bar chart representing the number of dysregulated genes post-SE and in chronic epilepsy. E, Venn diagram showing overlap of differentially expressed genes at the two time-points analyzed (post-SE and epilepsy). F, Comparison of differentially expressed genes post-SE and during epilepsy. The y-axis represents the FC enrichment and the $\mathrm{X}$-axis shows differently regulated genes post-SE ( 8 hours). Colors show the fold enrichment of differently regulated genes during epilepsy (14 days post-IAKA). One-sided Fisher exact test $(* * * P<.001)$

Grm5 transcripts, downregulated post-SE, were increased in mice treated with the CREB inhibitor 666-15 60 minutes after lorazepam administration when compared to vehicle-injected mice 8 hours post-SE (Figure 4C). Suggesting a functional role for CREB during IAKA-induced SE, mice treated with the CREB inhibitor 666-15 10 minutes prior to IAKA experienced less severe seizures during SE as evidenced by a reduction in behavioral seizures (Figure 4D), lower seizure total power (Figure 4E,F), and lower transcript level of the neuronal activity-regulated gene $c$-Fos post-SE (Figure 4G). In line with CREB inhibition reducing seizure severity during SE, HFHA spiking was slightly decreased in CREB inhibitor-treated mice (1032 \pm 251.5 seconds, vehicle, $\mathrm{n}=7$ vs $563.9 \pm 206.9$ seconds, CREB inhibitor, $\mathrm{n}=9 ; P=.16)$.

Thus, our results demonstrate that the IAKA model is a valid model to screen for and test novel anticonvulsive and antiepileptic drugs.

\section{4 | DISCUSSION}

Here, we demonstrate that the IAKA mouse model closely mimics molecular changes in the brain of patients with TLE once epilepsy is established, and therefore represents a valid model for the testing of novel antiepileptogenic drug targets.

A first result of our microarray analysis is that gene expression changes are much more prominent post-SE when compared to epilepsy, with functional profiles being broadly unique to each condition, in line with previous studies using models of SE induced via other chemoconvulsants. ${ }^{8,10}$ Thus, these findings confirm the importance of transcriptional changes during the initial insult, which may initiate a cascade of pathological changes leading eventually to the development of epilepsy. The cause of this initial surge in gene expression changes is most likely the increased neuronal excitability experienced during SE leading to the initiation of extensive neuronal death and gliosis. Why SE and epilepsy lead to the dysregulation of a different gene pool remains elusive. The most obvious explanations include differences in seizure severities, time-point of tissue analysis relative to seizures, and underlying pathology (ie, acute neurodegeneration vs chronically diseased brain). Interestingly, genes dysregulated during both conditions are either up- or downregulated, suggesting some common regulatory mechanisms during SE and epilepsy.

Our pathway analysis of genes dysregulated in the IAKA model post-SE showed in particular genes linked to signaling pathways enriched among upregulated genes and genes associated with metabolism and synaptic transmission among downregulated genes. This is in line with other models (eg, MAPK family) ${ }^{6,10}$ and suggests different stimuli activating similar pathways. During epilepsy, pathways most affected in the IAKA model included waste removal (upregulated) and synaptic transmission (downregulated). In particular, genes associated with lysosome activity were among the most affected genes, in line with genes linked to the mTOR pathway being commonly dysregulated during epilepsy. ${ }^{8,10}$ Of note, autophagic/lysosomal-related proteins are increasingly recognized to play an important role during epilepsy, with drugs targeting autophagy reported to modulate seizures. ${ }^{41}$

Although we have amassed many data on gene expression in different models, there has been a paucity of cross-model comparisons. Here, we compared our findings in the IAKA mouse model with two commonly used mouse models to induce SE and epilepsy (ie, IHKA and i.p. pilocarpine). The main finding here was that gene expression changes are much more similar between both KA mouse models when compared to the pilocarpine model. An unexpected result was the almost complete overlap between both KA models, post-SE and during epilepsy. This is even more remarkable, as both models display different pathologies during and post-SE. This includes severe seizures during IAKA-induced SE with cell death mainly restricted to the ipsilateral CA3 subfield versus mostly nonconvulsive SE and widespread hippocampal neurodegeneration in the IHKA mouse model. ${ }^{17}$ In contrast, when we compared the IAKA model with the i.p. pilocarpine mouse model, similarities were less obvious, in particular post-SE. Thus, gene expression changes seem to be more dependent on the chemoconvulsant 


\section{Comparison of gene expression changes}

A

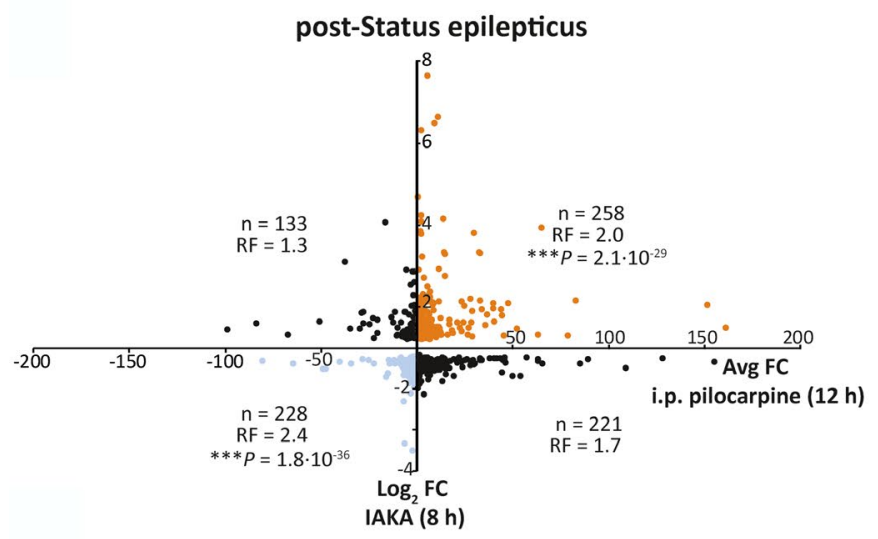

IAKA vs. i.p. pilocarpine (mouse model)
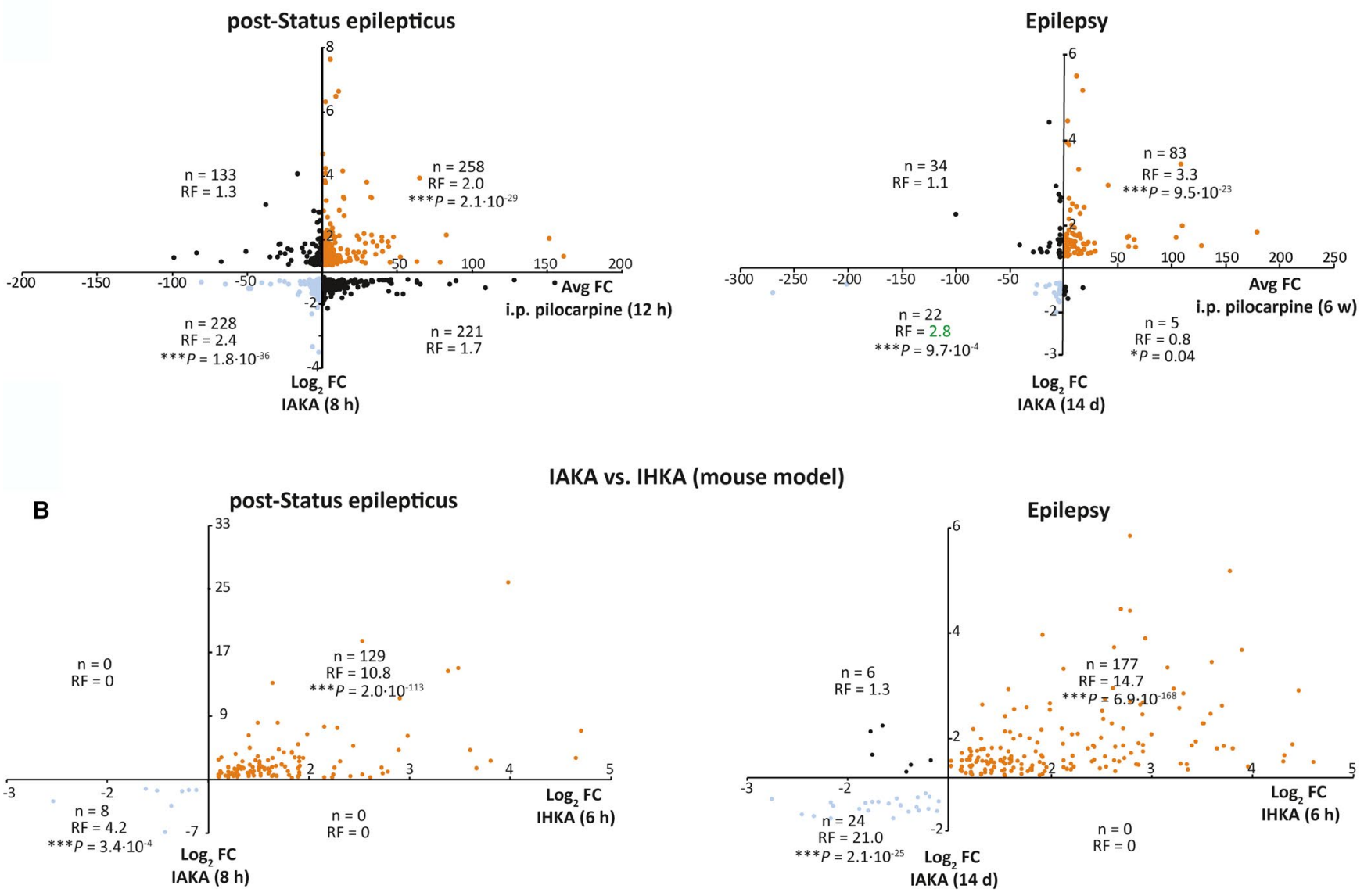

IAKA mouse model vs. TLE patients

C

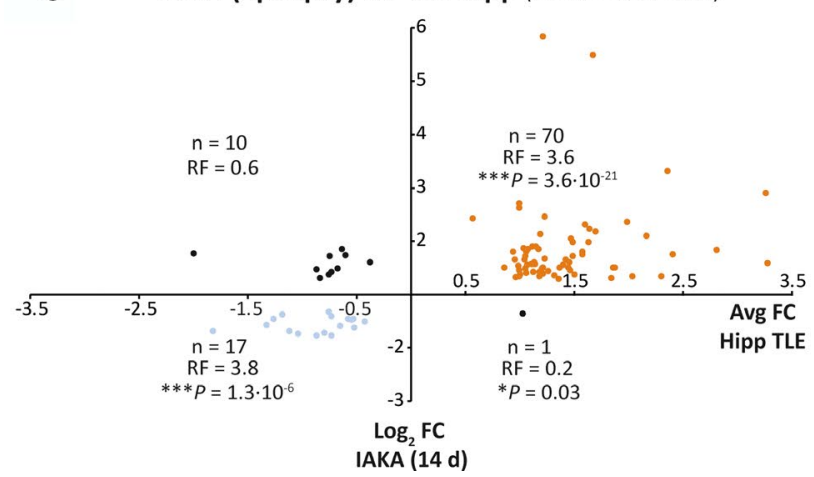

IAKA (epilepsy) vs. mTLE Cx (Guelfi et al. 2019)

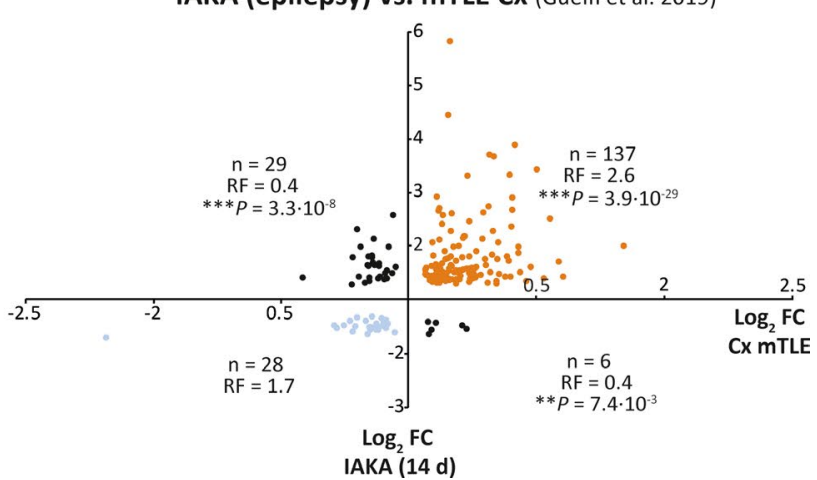

F I G URE 2 Comparison of genes undergoing expression changes in experimental models of epilepsy and temporal lobe epilepsy (TLE) patients. A, B, Graphs showing comparison of dysregulated genes in the hippocampus of (A) mice subjected to intra-amygdala kainic acid (IAKA) and intraperitoneal (i.p.) pilocarpine and (B) mice subjected to IAKA and intrahippocampal kainic acid (IHKA) after status epilepticus and during chronic epilepsy. C, Comparison of dysregulated genes in the hippocampus of IAKA-treated mice (14 days) and hippocampi (Hipp) from patients with TLE (left panel) and cortex (Cx) from patients with mesial TLE (mTLE; right panel). Upregulated genes are presented in orange in both conditions and downregulated genes are presented in blue. Overlap: representation factor (RF) $>2$ and $P<.05$, and dissimilar RF $<0.5$ and $P<.05$ by hypergeometric test. FC, fold change. $* * * P<.001$

used than pathology. It is, however, important to keep in mind that pilocarpine was delivered i.p., in contrast to the intracerebral delivery of KA.
One of the main results of our study is the close correlation of gene expression in the KA models when compared to human TLE, which reinforces the rationale for using these 


\section{GO terms of differentially expressed genes - KEGG Pathways}

A

post-Status epilepticus

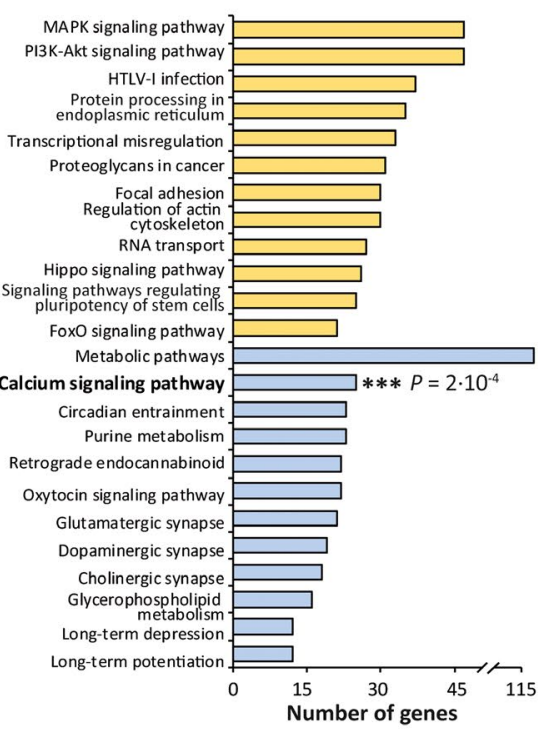

IAKA mouse model

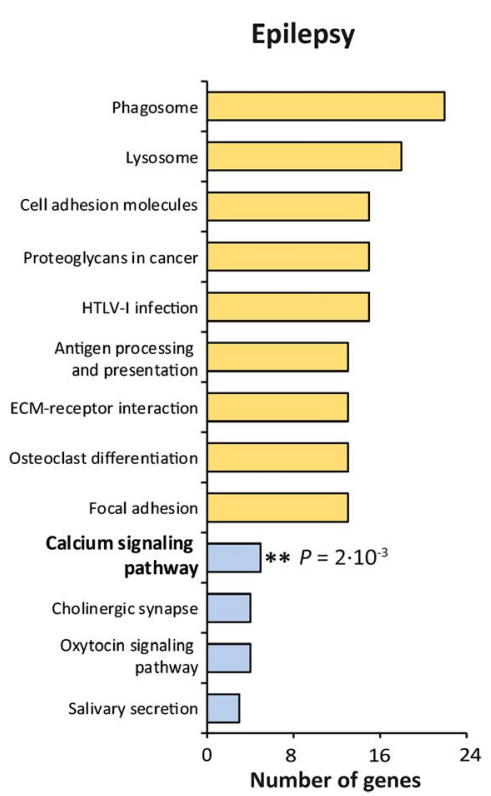

IAKA mouse model

B and TLE patients

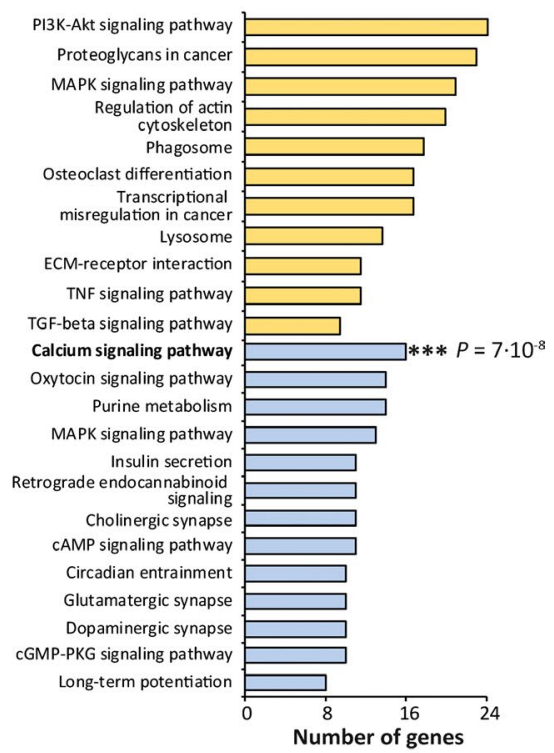

Validation via qRT-PCR

C Calcium signaling pathway (downregulated genes)

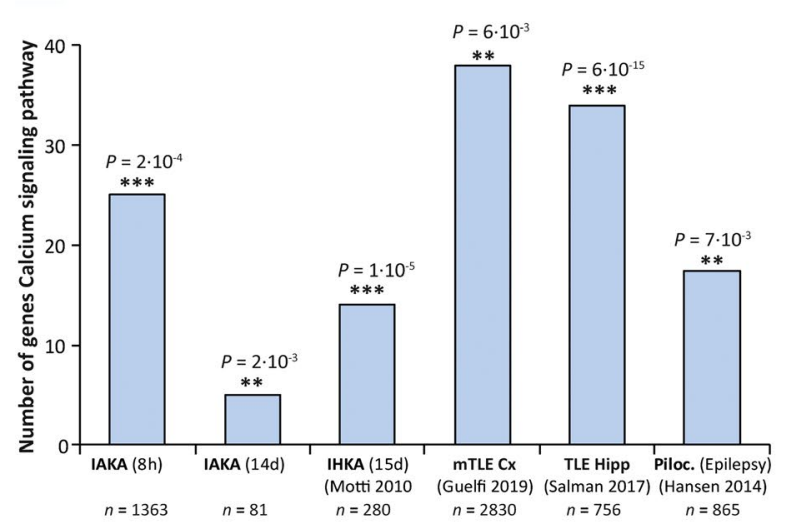

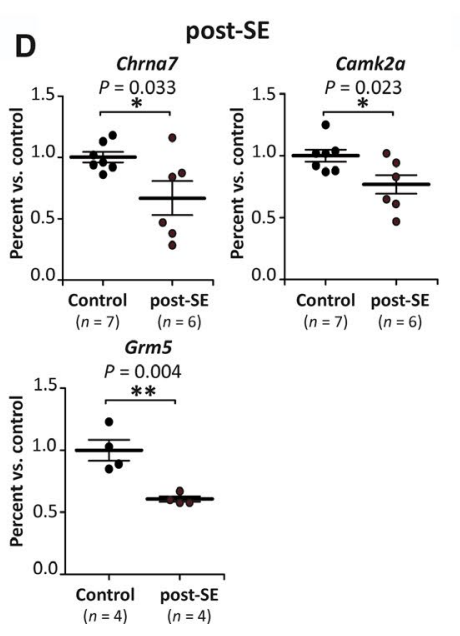

E

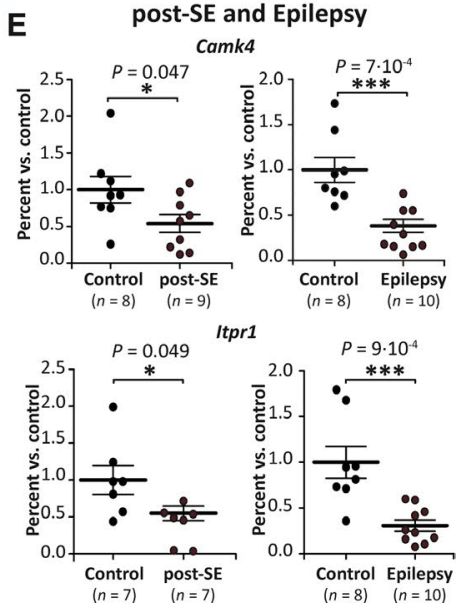

F I G U RE 3 Gene Ontology (GO) analysis and validation of microarray results. A, B, GO terms of (A) differently regulated genes after status epilepticus (SE) and during chronic epilepsy in the intra-amygdala kainic acid (IAKA) mouse model and (B) common dysregulated genes between temporal lobe epilepsy (TLE) patients and IAKA-treated epileptic mice. GO terms of up- and downregulated genes are presented in yellow and blue, respectively. $\mathrm{C}$, Number of genes associated with calcium signaling among downregulated genes in the IAKA and intrahippocampal kainic acid (IHKA) mouse models of epilepsy, TLE patients (hippocampus [Hippo] and cortex [Cx]) and the intraperitoneal pilocarpine (Piloc.) model of epilepsy (one-sided Fisher exact test). D, E, mRNA level analysis by quantitative real-time polymerase chain reaction (qRT-PCR) of downregulated genes involved in calcium signaling, (D) following SE (control $\mathrm{n}=7$ [Chrna7 and Camk2a] and 4 [Grm5], post-SE $\mathrm{n}=6$ [Chrna7 and Camk2a] and 4 [Grm5]) and (E) at both time-points (post-SE: control $\mathrm{n}=8$ [CamK4] and 7 [Itprl], post-SE $\mathrm{n}=9$ [CamK4] and 7 [Itpr 1]; epilepsy; control $\mathrm{n}=8$, epilepsy $\mathrm{n}=10$ ). Data are mean \pm standard error of the mean. $* P<.05, * * P<.01, * * * P<.001$ by two-sided unpaired $t$ test. mTLE, mesial TLE

models to identify and test novel antiepileptogenic drugs. These similarities are restricted to established epilepsy and partly lost when we compared human tissue with mouse tissue collected shortly following SE, suggesting established epilepsy in mice is a better model for TLE. Why the pilocarpine model shows fewer similarities with human TLE remains unknown. The pilocarpine model is, however, associated with peripheral immune responses prior to the induction of SE and most likely reflects a mixture of an ischemic and excitotoxic insult. ${ }^{42}$ In line with a strong overlap in gene expression between the IAKA model and TLE patients, GO term analysis of common dysregulated genes revealed similar 
TA B L E 1 Calcium signaling (downregulated genes): mouse models of epilepsy and/or TLE patients

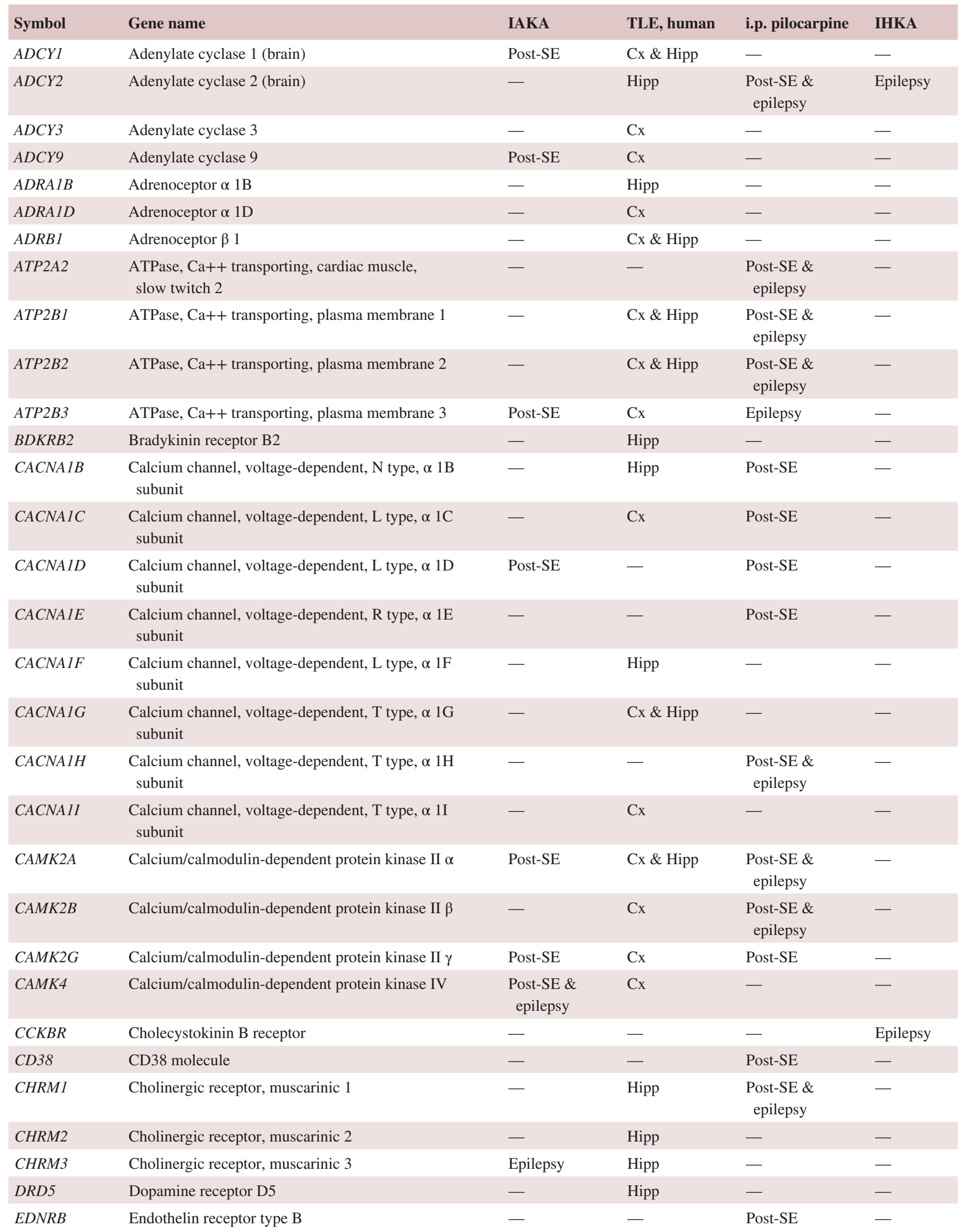


T A B L E 1 (Continued)

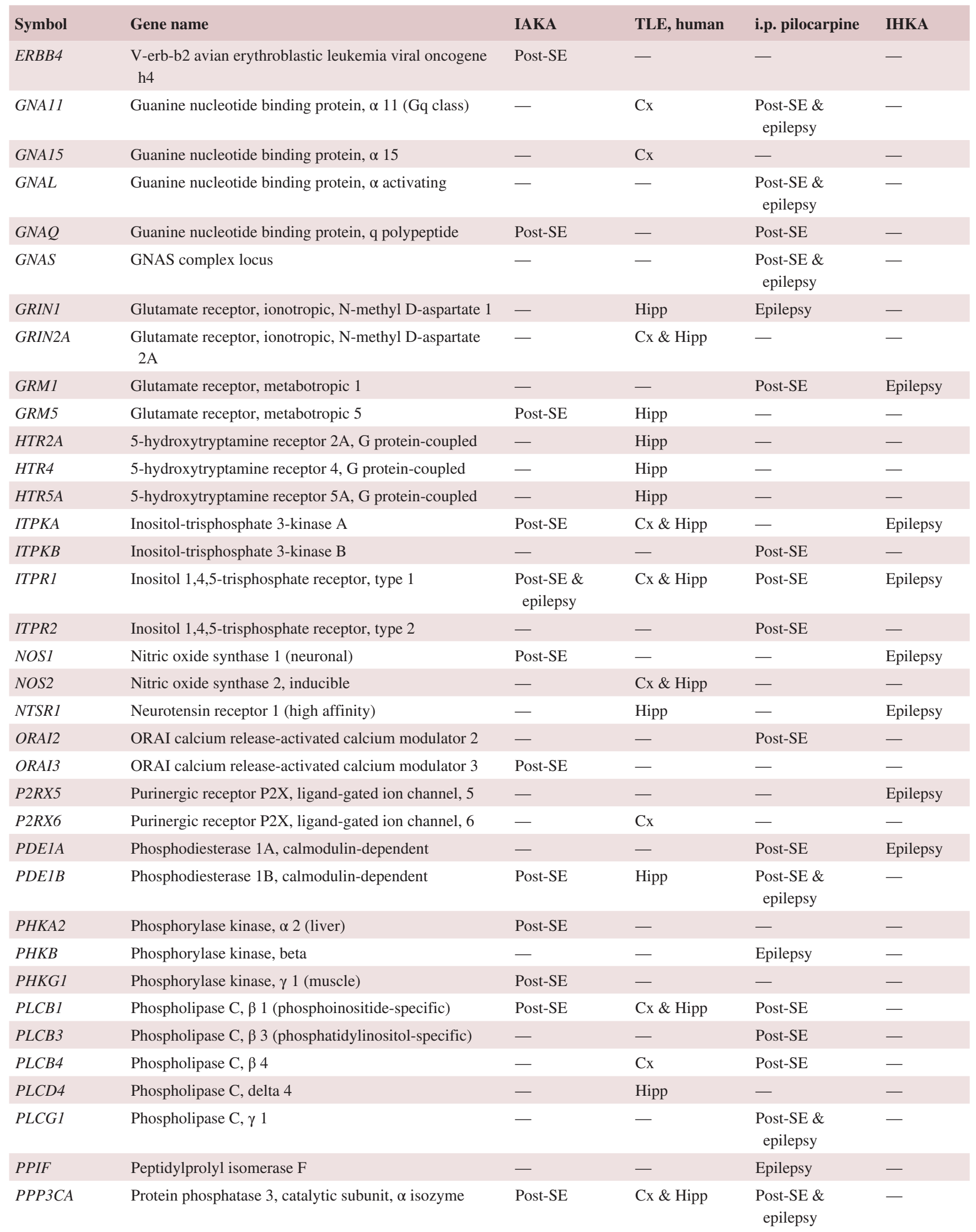


TABLE 1 (Continued)

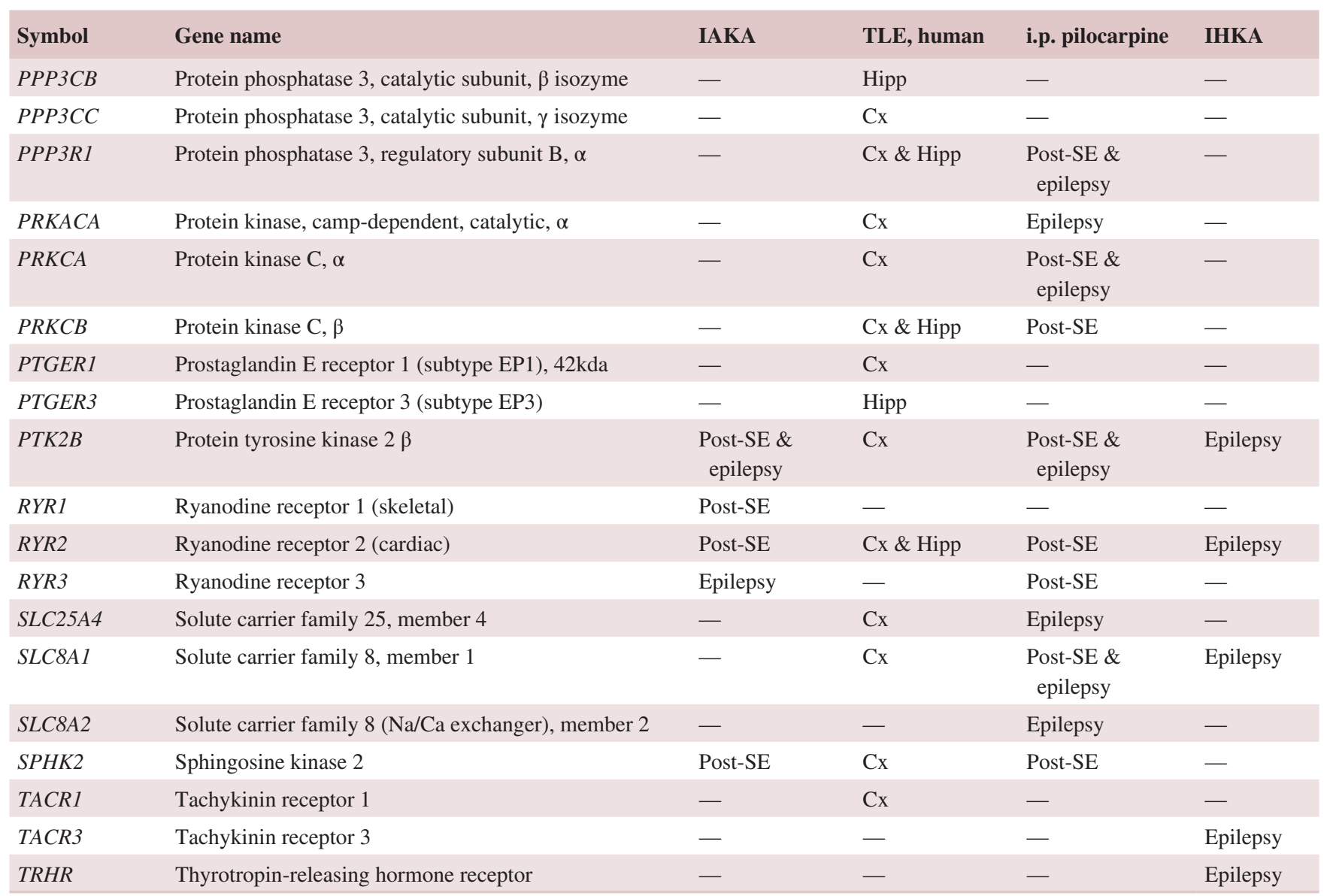

Abbreviations: Cx, cortex; Hipp, hippocampus; i.p., intraperitoneal; IAKA, intra-amygdala kainic acid; IHKA, intrahippocampal kainic acid; SE, status epilepticus; TLE, temporal lobe epilepsy.

pathways affected. Most notably, calcium signaling is one of the most consistently affected pathways across models and patients, which is consistent with previous reports. ${ }^{6,43,44}$ The effect of suppressed calcium signaling on seizures and epilepsy remains to be fully established. Calcium channels are implicated in numerous cellular processes, including synaptic plasticity, intracellular signal propagation, and the release of neurotransmitters. ${ }^{45}$ Data from knockout models of various calcium channels suggest the suppression of calcium signaling to be proepileptic (eg, ITPRI mutations). ${ }^{45,46}$ The suppression of calcium signaling has, however, also been suggested as a protective mechanism reducing synaptic

F I G U RE 4 CREB1 as upstream transcription factor during status epilepticus and epilepsy. A, Prediction of transcription factors involved in gene upregulation (yellow bars) and downregulation (blue bars) after status epilepticus (SE) and during epilepsy by using Ingenuity Pathway Analysis (IPA). Of note, the transcription factor CREB1 is one of the transcription factors with the highest number of predicted target genes among the up- and downregulated gene pool during both conditions, post-SE and during epilepsy. B, Schematic of experimental design to test CREB inhibition on seizure severity during SE and gene expression. To test effects of CREB inhibition on seizure severity during SE, mice were treated intracerebroventricularly (i.c.v.) with the CREB inhibitor 666-15 (2 nmol) 10 minutes before intra-amygdala kainic acid (IAKA) injection. To test the effects of CREB inhibition on gene expression, mice were treated i.c.v. with the CREB inhibitor 666-15 (2 nmol) 60 minutes after lorazepam treatment. C, Graphs showing increased mRNA levels of the two CREB target genes, CamK4 and Grm5, in mice treated with the CREB inhibitor 666-15 60 minutes after lorazepam injection (vehicle, $\mathrm{n}=4$; CREB inhibitor [Inhib.], $\mathrm{n}=5$ ). D, Behavioral severity of seizures (mean Racine score) scored every 5 minutes and total score, in mice subjected to IAKA treated with vehicle $(n=6)$ and treated with the CREB inhibitor 666-15 10 minutes before IAKA $(n=6)$. E, Representative electroencephalographic (EEG) recordings presented as heat maps of frequency and amplitude data showing reduced seizure severity in mice treated with the CREB inhibitor (Creb Inh) 666-15 10 minutes prior to IAKA during a 40-minute recording period starting at the time of IAKA injection. KA, kainic acid; Veh, vehicle. F, Bar chart showing decreased EEG total power during SE in mice treated with the CREB inhibitor 666-15 10 minutes before IAKA $(n=9)$ when compared to vehicle-treated mice $(n=7)$. G, Reduced transcript levels of the neuronal activity-regulated gene $c$-Fos in mice treated with the CREB inhibitor 666-15 10 minutes before IAKA when compared to vehicle-treated mice 8 hours after lorazepam injection ( $\mathrm{n}=5$ per group). C, D, F, G, Two-sided unpaired $t$ test. Data are mean \pm standard error of the mean. $* P<.05$, $* * P<.01$, *** $P<.001$. 
A

Upstream transcription factors (IPA ${ }^{\circledR}$ analysis)
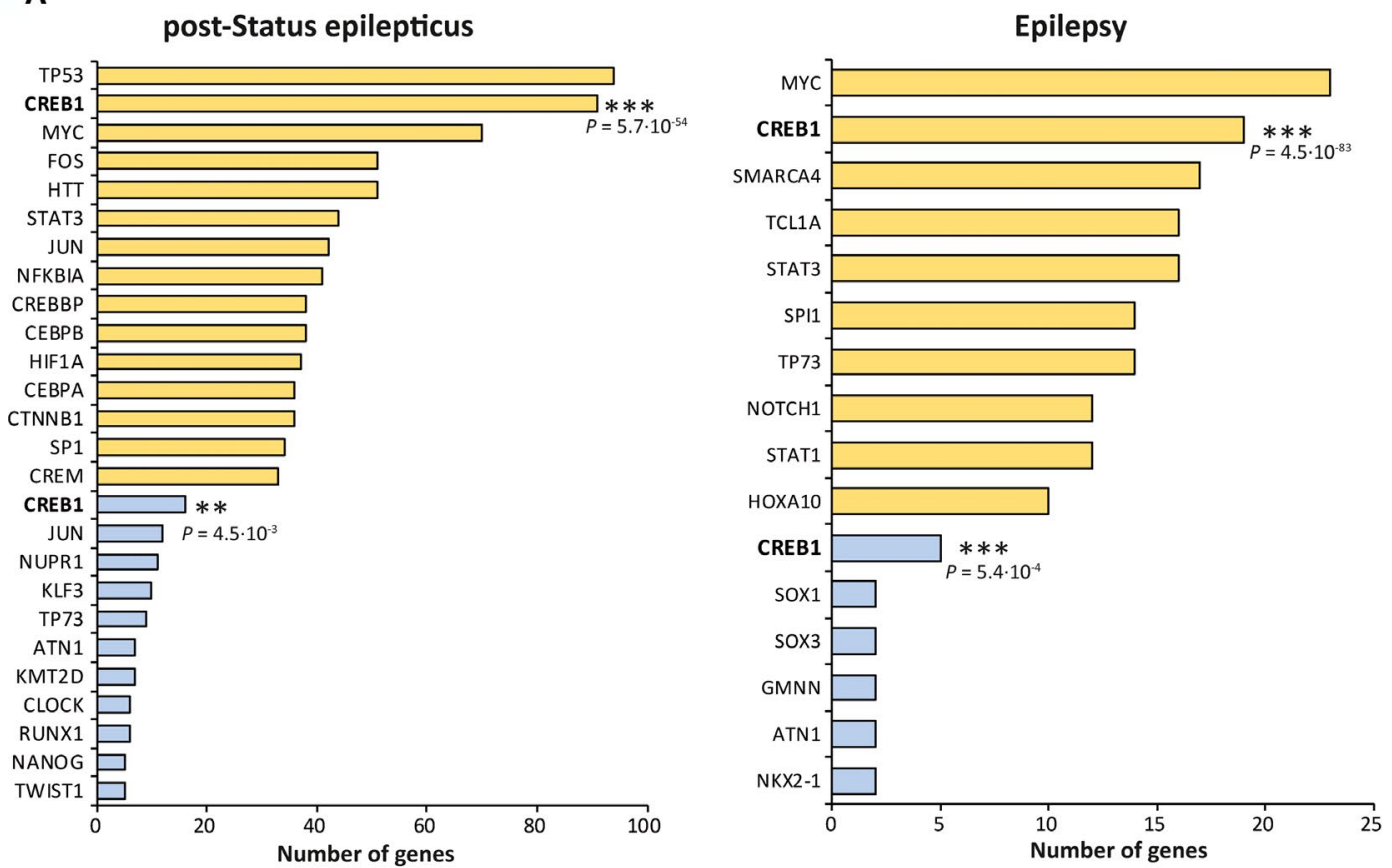

B

Experimental design
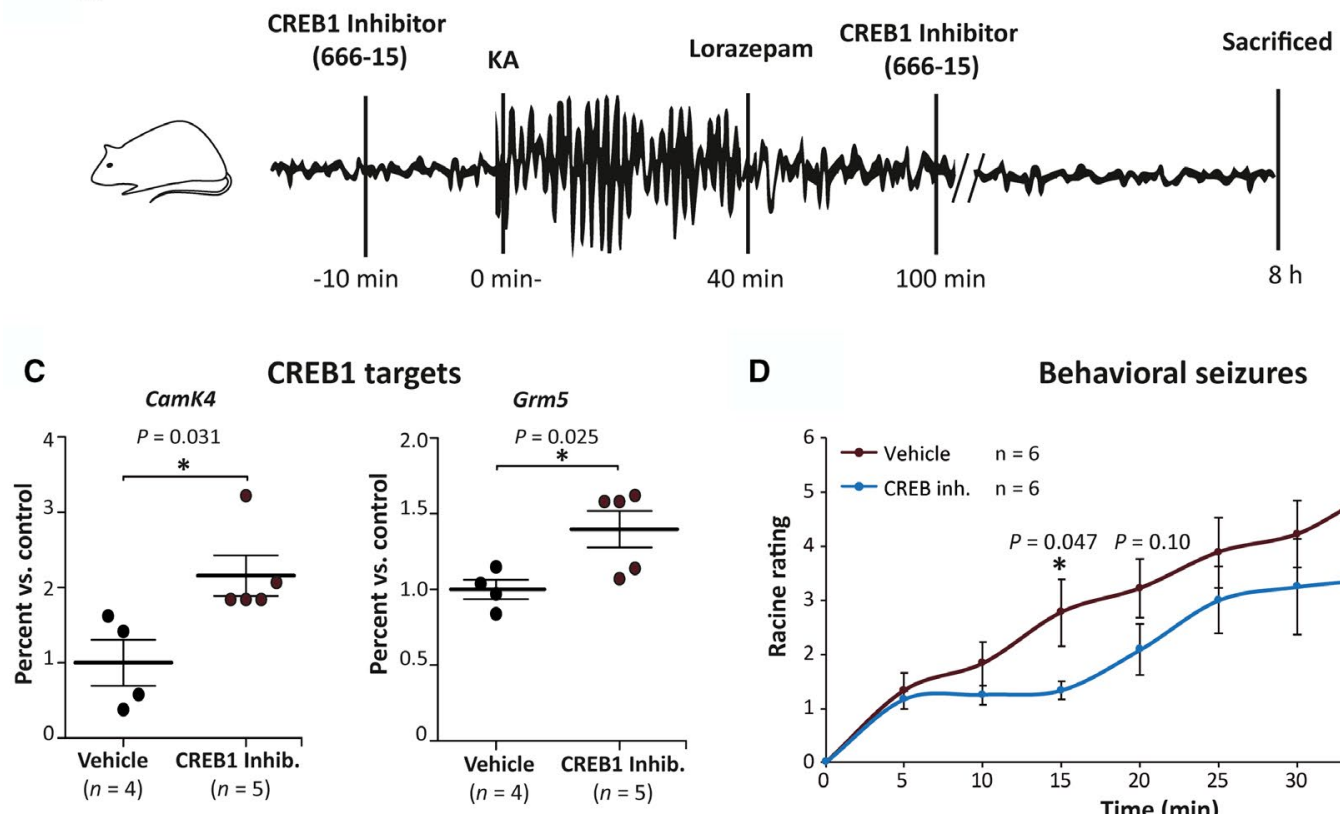

D

Behavioral seizures
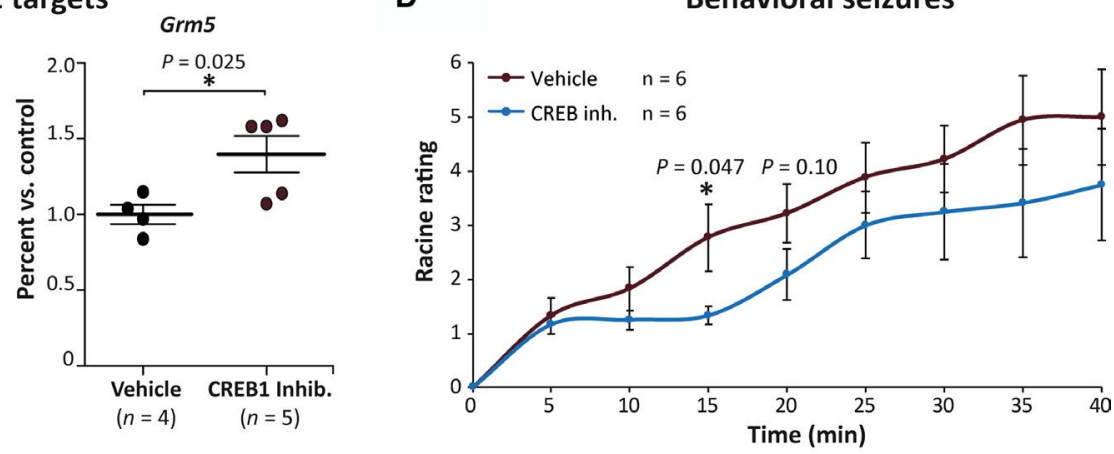

E

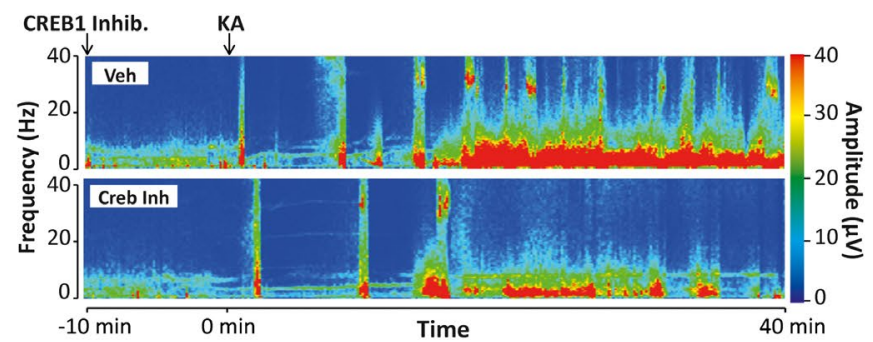

F $\quad$ EEG

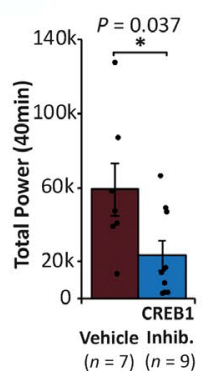

G c-Fos (mRNA)

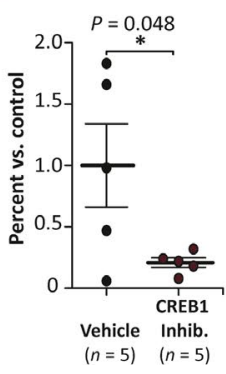


transmission and thereby hyperexcitability in brain tissue. Calcium signaling was suppressed in mice undergoing epileptic preconditioning, ${ }^{24}$ and several ASDs are suggested to act, at least partly, via the suppression of calcium channels (eg, levetiracetam, valproate, phenytoin). ${ }^{45}$

Finally, our IPA analysis predicted the protein CREB1 as one of the main upstream transcription factors regulating gene transcription during SE and epilepsy. This is in line with previous findings identifying CREB as one of the main transcription factors in the pilocarpine mouse model. ${ }^{10} \mathrm{~A}$ role for CREB during seizures has been previously demonstrated, with decreased levels of CREB leading to seizure reduction following pilocarpine in mice. ${ }^{47}$ However, whether CREB contributes to seizures via the regulation of calcium signaling warrants further investigation. Interestingly, 553 was predicted as one of the main transcription factors driving gene transcription post-SE. p53 has previously been linked to seizure generation and cell death in the IAKA model. ${ }^{48}$ Although our IPA analysis has focused primarily on transcription factors, other mechanisms may also impact on mRNA levels, such as microRNAs or mRNA polyadenylation among many others. ${ }^{31,32,49}$

Possible limitations of our study include that gene expression has only been analyzed shortly post-SE and once epilepsy was already established, omitting the seizure-free latent period. The latent period in the IAKA mouse model is, however, almost absent, ${ }^{17}$ and the process of epileptogenesis is ongoing beyond the occurrence of the first epileptic seizure. ${ }^{5}$ A possible confounding factor between models is treatment with anticonvulsants post-SE. Whereas IAKA-treated mice received the anticonvulsant lorazepam to stop/ameliorate SE, no anticonvulsant was used in the IHKA or the i.p. pilocarpine model. ${ }^{10,16,36}$ The closer correlation between both KA models when compared to the pilocarpine model suggests, however, the trigger of SE being more important than anticonvulsant treatment. Moreover, expression profiles have been analyzed using different analysis platforms (eg, microarray vs sequencing), and cutoffs for analysis might be different. In addition, time-points analyzed relative to initial insult differ between animal models. We have, however, taken into account the different timelines of each model (eg, longer latent period in the pilocarpine model compared to the IAKA model). ${ }^{16,42}$ Finally, some changes may have been masked by analyzing the whole hippocampus rather than subfields. Previous studies analyzing gene expression in TLE patients have, however, used resected hippocampal tissue regardless of subfields.

In summary, our results demonstrate the IAKA mouse model closely mimicking human TLE, thus providing strong rationale for using the IAKA model to identify potential targets for disease-modifying treatments.

\section{ACKNOWLEDGMENTS}

This work was supported by funding from the Health Research Board (HRA-POR-2015-1243 to T.E.); Science Foundation
Ireland (17/CDA/4708 to T.E.); the European Regional Development Fund; FutureNeuro industry partners (16/RC/3948 to D.C.H.); the H2020 Marie Skłodowska-Curie Actions Individual Fellowship (796600 to L.D.D.-G. and 753527 to E.B.); the European Union's Horizon 2020 research and innovation program under the Marie Skłodowska-Curie grant agreement (766124 to T.E.); Centro de Investigación Biomédica en Red de Enfermedades Neurodegenerativas (PI20152/06-3 and PI2018/06-1 to J.J.L); Ministerio de Economía y Competitividad (SAF2015-65371-R to J.J.L.); Ministerio de Ciencia, Innovación y Universidades (RTI2018-096322-B-I00 to J.J.L.) and a Spark grant (SNSF; CRSK-3_190764/1 to A.P.). We thank the following core facilities: IRB-Functional Genomic and IRB-Bioinformatics/Biostatistics.

\section{CONFLICT OF INTEREST}

None of the authors has any conflict of interest to disclose.

\section{ETHICAL PUBLICATION STATEMENT}

We confirm that we have read the Journal's position on issues involved in ethical publication and affirm that this report is consistent with those guidelines.

\section{ORCID}

Giorgia Conte (iD https://orcid.org/0000-0003-0566-9339

Alberto Parras (D) https://orcid.org/0000-0003-0999-2600

David C. Henshall iD https://orcid.

org/0000-0001-6237-9632

Tobias Engel (D) https://orcid.org/0000-0001-9137-0637

\section{REFERENCES}

1. Bialer M, White HS. Key factors in the discovery and development of new antiepileptic drugs. Nat Rev Drug Discov. 2010;9:68-82.

2. Moshé SL, Perucca E, Ryvlin P, Tomson T. Epilepsy: new advances. Lancet. 2015;385:884-98.

3. Thijs RD, Surges R, O'Brien TJ, Sander JW. Epilepsy in adults. Lancet. 2019;393:689-701.

4. Chang BS, Lowenstein DH. Epilepsy. N Engl J Med. 2003;349:1257-66.

5. Pitkanen A, Lukasiuk K. Mechanisms of epileptogenesis and potential treatment targets. Lancet Neurol. 2011;10:173-86.

6. Gorter JA, van Vliet EA, Aronica E, Breit T, Rauwerda H, Lopes da Silva FH, et al. Potential new antiepileptogenic targets indicated by microarray analysis in a rat model for temporal lobe epilepsy. J Neurosci. 2006;26:11083-110.

7. Wang YY, Smith P, Murphy M, Cook M. Global expression profiling in epileptogenesis: does it add to the confusion? Brain Pathol. 2010;20:1-16.

8. Okamoto OK, Janjoppi L, Bonone FM, Pansani AP, da Silva AV, Scorza FA, et al. Whole transcriptome analysis of the hippocampus: toward a molecular portrait of epileptogenesis. BMC Genomics. 2010;11:230.

9. Goldberg EM, Coulter DA. Mechanisms of epileptogenesis: a convergence on neural circuit dysfunction. Nat Rev Neurosci. 2013;14:337-49. 
10. Hansen KF, Sakamoto K, Pelz C, Impey S, Obrietan K. Profiling status epilepticus-induced changes in hippocampal RNA expression using high-throughput RNA sequencing. Sci Rep. 2014;4:6930.

11. Dixit AB, Banerjee J, Srivastava A, Tripathi M, Sarkar C, Kakkar A, et al. RNA-seq analysis of hippocampal tissues reveals novel candidate genes for drug refractory epilepsy in patients with MTLE-HS. Genomics. 2016;107:178-88.

12. Salman MM, Sheilabi MA, Bhattacharyya D, Kitchen P, Conner AC, Bill RM, et al. Transcriptome analysis suggests a role for the differential expression of cerebral aquaporins and the MAPK signalling pathway in human temporal lobe epilepsy. Eur J Neurosci. 2017;46:2121-32.

13. Srivastava PK, van Eyll J, Godard P, Mazzuferi M, DelahayeDuriez A, Van Steenwinckel J, et al. A systems-level framework for drug discovery identifies Csf1R as an anti-epileptic drug target. Nat Commun. 2018;9:3561.

14. Guelfi S, Botia JA, Thom M, Ramasamy A, Perona M, Stanyer L, et al. Transcriptomic and genetic analyses reveal potential causal drivers for intractable partial epilepsy. Brain. 2019;142:1616-30.

15. Loscher W. Animal models of seizures and epilepsy: past, present, and future role for the discovery of antiseizure drugs. Neurochem Res. 2017;42:1873-88.

16. Mouri G, Jimenez-Mateos E, Engel T, Dunleavy M, Hatazaki S, Paucard A, et al. Unilateral hippocampal CA3-predominant damage and short latency epileptogenesis after intra-amygdala microinjection of kainic acid in mice. Brain Res. 2008;1213:140-51.

17. Welzel L, Schidlitzki A, Twele F, Anjum M, Loscher W. A face-toface comparison of the intra-amygdala and intrahippocampal kainate mouse models of mesial temporal lobe epilepsy and their utility for testing novel therapies. Epilepsia. 2020;61:157-70.

18. Wu YP, Siao C-J, Lu W, Sung T-C, Frohman MA, Milev P, et al. The tissue plasminogen activator (tPA)/plasmin extracellular proteolytic system regulates seizure-induced hippocampal mossy fiber outgrowth through a proteoglycan substrate. J Cell Biol. 2000;148:1295-304.

19. Li T, Ren G, Lusardi T, Wilz A, Lan JQ, Iwasato T, et al. Adenosine kinase is a target for the prediction and prevention of epileptogenesis in mice. J Clin Invest. 2008;118:571-82.

20. Jimenez-Mateos EM, Engel T, Merino-Serrais P, McKiernan RC, Tanaka K, Mouri G, et al. Silencing microRNA-134 produces neuroprotective and prolonged seizure-suppressive effects. Nat Med. 2012;18:1087-94.

21. Liu G, Gu B, He X-P, Joshi R, Wackerle H, Rodriguiz R, et al. Transient inhibition of TrkB kinase after status epilepticus prevents development of temporal lobe epilepsy. Neuron. 2013;79:31-8.

22. Iori V, Iyer AM, Ravizza T, Beltrame L, Paracchini L, Marchini $\mathrm{S}$, et al. Blockade of the IL-1R1/TLR4 pathway mediates disease-modification therapeutic effects in a model of acquired epilepsy. Neurobiol Dis. 2017;99:12-23.

23. Colasante G, Qiu Y, Massimino L, Di Berardino C, Cornford JH, Snowball A, et al. In vivo CRISPRa decreases seizures and rescues cognitive deficits in a rodent model of epilepsy. Brain. 2020;143:891-905.

24. Jimenez-Mateos EM, Hatazaki S, Johnson MB, Bellver-Estelles C, Mouri G, Bonner C, et al. Hippocampal transcriptome after status epilepticus in mice rendered seizure damage-tolerant by epileptic preconditioning features suppressed calcium and neuronal excitability pathways. Neurobiol Dis. 2008;32:442-53.
25. Engel T, Sanz-Rodgriguez A, Jimenez-Mateos EM, Concannon CG, Jimenez-Pacheco A, Moran C, et al. CHOP regulates the p53MDM2 axis and is required for neuronal survival after seizures. Brain. 2013;136:577-92.

26. Alves M, De Diego GL, Conte G, Jimenez-Mateos EM, D'Orsi B, Sanz-Rodriguez A, et al. Context-specific switch from anti- to pro-epileptogenic function of the $\mathrm{P} 2 \mathrm{Y} 1$ receptor in experimental epilepsy. J Neurosci. 2019;39:5377-92.

27. Araki T, Simon RP, Taki W, Lan JQ, Henshall DC. Characterization of neuronal death induced by focally evoked limbic seizures in the C57BL/6 mouse. J Neurosci Res. 2002;69:614-21.

28. Jimenez-Pacheco A, Mesuret G, Sanz-Rodriguez A, Tanaka K, Mooney $\mathrm{C}$, Conroy $\mathrm{R}$, et al. Increased neocortical expression of the P2X7 receptor after status epilepticus and anticonvulsant effect of P2X7 receptor antagonist A-438079. Epilepsia. 2013;54:1551-61.

29. Xie F, Li BX, Kassenbrock A, Xue C, Wang X, Qian DZ, et al. Identification of a potent inhibitor of CREB-mediated gene transcription with efficacious in vivo anticancer activity. J Med Chem. 2015;58:5075-87.

30. Li BX, Gardner R, Xue C, Qian DZ, Xie F, Thomas G, et al. Systemic inhibition of CREB is well-tolerated in vivo. Sci Rep. 2016;6:34513.

31. Parras A, Anta H, Santos-Galindo M, Swarup V, Elorza A, NietoGonzález JL, et al. Autism-like phenotype and risk gene mRNA deadenylation by CPEB4 mis-splicing. Nature. 2018;560:441-6.

32. Parras A, de Diego-Garcia L, Alves M, Beamer E, Conte G, Jimenez-Mateos EM, et al. Polyadenylation of mRNA as a novel regulatory mechanism of gene expression in temporal lobe epilepsy. Brain. 2020;143(7):2139-53.

33. Gentleman RC, Carey VJ, Bates DM, Bolstad B, Dettling M, Dudoit S, et al. Bioconductor: open software development for computational biology and bioinformatics. Genome Biol. 2004;5:R80.

34. Ritchie ME, Phipson B, Wu DI, Hu Y, Law CW, Shi W, et al. limma powers differential expression analyses for RNA-sequencing and microarray studies. Nucleic Acids Res. 2015;43(7):e47.

35. Fury W, Batliwalla F, Gregersen PK, Li W. Overlapping probabilities of top ranking gene lists, hypergeometric distribution, and stringency of gene selection criterion. Conf Proc IEEE Eng Med Biol Soc. 2006;2006:5531-4.

36. Motti D, Le Duigou C, Eugène E, Chemaly N, Wittner L, Lazarevic $\mathrm{D}$, et al. Gene expression analysis of the emergence of epileptiform activity after focal injection of kainic acid into mouse hippocampus. Eur J Neurosci. 2010;32:1364-79.

37. da Huang W, Sherman BT, Lempicki RA. Systematic and integrative analysis of large gene lists using DAVID bioinformatics resources. Nat Protoc. 2009;4:44-57.

38. Kramer A, Green J, Pollard J Jr, Tugendreich S. Causal analysis approaches in Ingenuity Pathway Analysis. Bioinformatics. 2014;30:523-30.

39. Alves M, Kenny A, de Leo G, Beamer EH, Engel T. Tau phosphorylation in a mouse model of temporal lobe epilepsy. Front Aging Neurosci. 2019;11:308.

40. Huang C, Chi X-S, Li R, Hu X, Xu H-X, Li J-M, et al. Inhibition of P2X7 receptor ameliorates nuclear factor-kappa B mediated neuroinflammation induced by status epilepticus in rat hippocampus. $\mathrm{J}$ Mol Neurosci. 2017;63:173-84.

41. Fassio A, Falace A, Esposito A, Aprile D, Guerrini R, Benfenati F. Emerging role of the autophagy/lysosomal degradative pathway in 
neurodevelopmental disorders with epilepsy. Front Cell Neurosci. 2020;14:39.

42. Curia G, Longo D, Biagini G, Jones RS, Avoli M. The pilocarpine model of temporal lobe epilepsy. J Neurosci Methods. 2008;172:143-57.

43. Elliott RC, Miles MF, Lowenstein DH. Overlapping microarray profiles of dentate gyrus gene expression during development- and epilepsy-associated neurogenesis and axon outgrowth. J Neurosci. 2003;23:2218-27.

44. Lauren HB, Lopez-Picon FR, Brandt AM, Rios-Rojas CJ, Holopainen IE. Transcriptome analysis of the hippocampal CA1 pyramidal cell region after kainic acid-induced status epilepticus in juvenile rats. PLoS One. 2010;5(5):e10733.

45. Rajakulendran S, Hanna MG. The role of calcium channels in epilepsy. Cold Spring Harb Perspect Med. 2016;6:a022723.

46. Matsumoto M, Nakagawa T, Inoue T, Nagata E, Tanaka K, Takano $\mathrm{H}$, et al. Ataxia and epileptic seizures in mice lacking type 1 inositol 1,4,5-trisphosphate receptor. Nature. 1996;379:168-71.

47. Zhu X, Han X, Blendy JA, Porter BE. Decreased CREB levels suppress epilepsy. Neurobiol Dis. 2012;45:253-63.

48. Engel T, Tanaka K, Jimenez-Mateos EM, Caballero-Caballero A, Prehn JH, Henshall DC. Loss of p53 results in protracted electrographic seizures and development of an aggravated epileptic phenotype following status epilepticus. Cell Death Dis. 2010;1(10):e79.

49. Henshall DC, Hamer HM, Pasterkamp RJ, Goldstein DB, Kjems J, Prehn JHM, et al. MicroRNAs in epilepsy: pathophysiology and clinical utility. Lancet Neurol. 2016;15:1368-76.

\section{SUPPORTING INFORMATION}

Additional supporting information may be found online in the Supporting Information section.

How to cite this article: Conte G, Parras A, Alves M, et al. High concordance between hippocampal transcriptome of the mouse intra-amygdala kainic acid model and human temporal lobe epilepsy. Epilepsia. 2020;61:2795-2810. https://doi.org/10.1111/epi.16714 